\title{
Theoretic Criteria for Antibody Penetration into Solid Tumors and Micrometastases
}

\author{
Greg M. Thurber ${ }^{1}$, Stefan C. Zajic ${ }^{1}$, and K. Dane Wittrup ${ }^{1,2}$ \\ ${ }^{I}$ Department of Chemical Engineering, Massachusetts Institute of Technology, Cambridge, Massachusetts; and ${ }^{2}$ Biological Engineering \\ Division, Massachusetts Institute of Technology, Cambridge, Massachusetts
}

\begin{abstract}
Targeting tumors with antibody-based therapeutics is a complex task presenting multiple kinetic barriers. Antibody internalization and clearance inhibit uptake both in solid tumors, limited by tumor vascular permeability, and in micrometastases, limited by diffusion. Methods: A modeling exercise is used to introduce 2 simple criteria that must be less than unity for saturation of both tumors and micrometastases. The clearance modulus and the Thiele modulus are ratios of the plasma clearance rate and antibody catabolism, respectively, to the tumor tissue penetration rate. Results: Even low rates of antigen internalization from constitutive membrane turnover can significantly retard antibody penetration. Rapid clearance of single-chain variable fragments also hinders uptake, often more than counterbalancing their more rapid extravasation and diffusion. Conclusion: The model illustrates that with the large resistance from the tumor capillary, antibodies may be more suitable for targeting micrometastases than vascularized tumors.
\end{abstract}

Key Words: molecular imaging; monoclonal antibodies; radionuclide therapy; biodistribution; penetration; pharmacokinetics

J Nucl Med 2007; 48:995-999

DOI: 10.2967/jnumed.106.037069

\section{A}

ntibodies and antibody fragments are promising agents for cancer detection and treatment. One of the major restrictions of these drugs for cancer therapy is poor transport, which results in low concentrations and a lack of targeting of all cells in a tumor (1). Delivery of antibodies to tumor cells is a complex task with many rates involved, as illustrated in Figure 1. Effective therapy requires treatment of both solid, vascularized tumors and micrometastases with different pharmacokinetic parameters.

Several different models have been developed to illustrate some fundamental trends in targeting. van Osdol et al. have shown that antibodies tend to exhibit a "binding site barrier" because of their clearance and penetration (2), and extensive work on fluid flow and macromolecular distribu-

\footnotetext{
Received Oct. 10, 2006; revision accepted Nov. 27, 2006.

For correspondence or reprints contact: K. Dane Wittrup, PhD, Department of Chemical Engineering and Biological Engineering Division, Massachusetts Institute of Technology, Building E19-551, 77 Massachusetts Ave., Cambridge, MA 02139.

E-mail: wittrup@mit.edu

COPYRIGHT @ 2007 by the Society of Nuclear Medicine, Inc.
}

tion has been performed by Jain (1). Using a simplified model, Graff and Wittrup highlighted the importance of antibody dose for saturating tissue and the necessity of high affinity for retention in the tumor (3).

Although providing useful insights, these prior models have not fully delineated the microscopic distribution effects of antibody catabolism. The turnover rate was not examined quantitatively, and one would expect differences between an antibody binding a receptor internalized by a clathrin-coated pit mechanism, constitutive membrane turnover, and extracellular matrix protein turnover. Internalization of ErbB receptor family members, targets for 2 antibody drugs approved by the Food and Drug Administration, is well characterized $(4,5)$. A multitude of other antibodies against various targets have also been shown to internalize at broadly varying rates $(6,7)$, demonstrating the need for an in-depth consideration of the effects of internalization on tumor targeting. This analysis reveals that internalization has the potential to severely limit the penetration of antibodies.

\section{MATERIALS AND METHODS}

We present 2 criteria to predict the extent of antibody targeting before and during saturation. The model is validated against published experimental data and is intended to provide a framework in which to analyze and interpret antibody targeting in vivo while also highlighting strategies for improvement in antibody micropharmacokinetics.

Dimensionless ratios of pairs of critical rates, sometimes referred to as moduli, simplify identification of the most dominant rates under particular conditions. We derive here 2 such moduli for tumor targeting.

\section{Clearance Modulus}

The clearance modulus, $\Gamma$, is a ratio of the time to saturate a given volume of tissue to the time that the antibody persists in the plasma. If the characteristic time for targeting a given radius is longer than the time the antibody is in the blood ( $\Gamma>1$ ), antibody will not reach this distance.

$$
\Gamma_{\text {tumor }} \equiv \frac{\ln (2) \cdot R^{2}\left([\mathrm{Ag}]_{t} / \varepsilon\right)}{2 \cdot[A b]_{\text {plasma }, 0} \cdot P \cdot R_{\text {cap }}\left(A \cdot t_{1 / 2, \alpha}+B \cdot t_{1 / 2, \beta}\right)} \quad \text { Eq. } 1
$$


FIGURE 1. Rate processes for tumor and micrometastasis targeting include blood clearance (1), extravasation (2), lymphatic clearance in normal tissue (3), diffusion into tumor tissue (4), binding (5), internalization (6), recycling (7), and degradation (8). The spheric (metastasis) and cylindric (tumor) radii $(\mathrm{R})$ are similar because of oxygen diffusion and consumption limitations but may differ slightly.

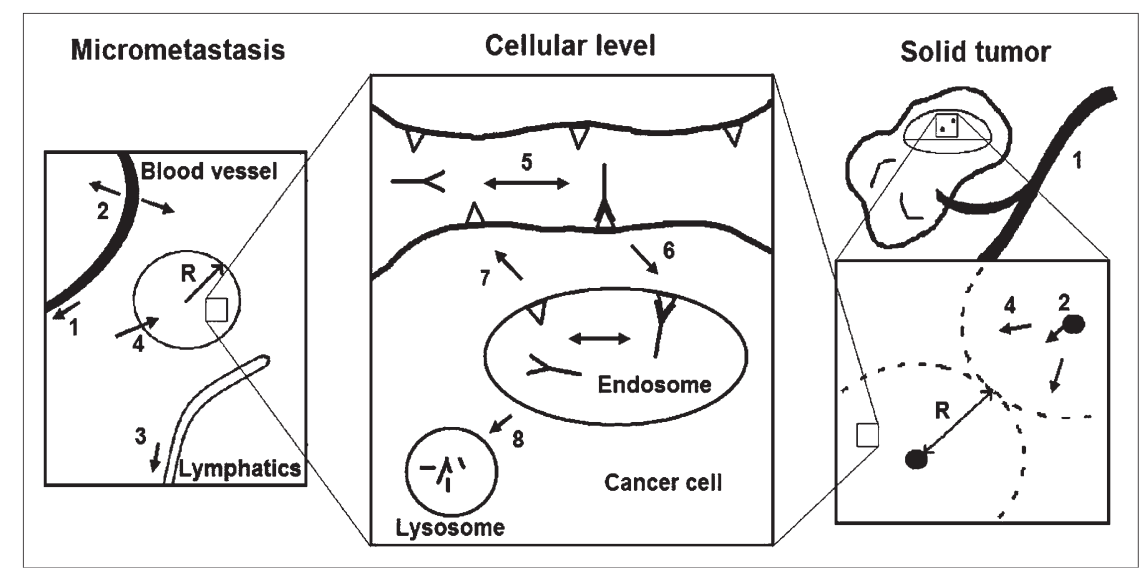

$$
\begin{aligned}
\Gamma_{m e t} & \equiv \frac{R^{2}\left([A g]_{t} / \varepsilon\right)}{6 D \cdot\left(A U C_{A b, \text { normal }}\right)} \\
& =\frac{\ln (2) \cdot R^{2}\left([A g]_{t} / \varepsilon\right)}{6 D \cdot\left([A b]_{\text {plasma }, 0}\left(\frac{\kappa}{\lambda}\right)\left(A \cdot t_{1 / 2, \alpha}+B \cdot t_{1 / 2, \beta}\right)\right)}
\end{aligned}
$$

The $R^{2}$ term defines the volume of tissue considered in a Kroghlike cylinder or spheric metastasis, $[\mathrm{Ag}]_{t}$ is the antigen concentration in the overall tumor volume, and $\varepsilon$ is the effective void fraction. $[A b]_{\text {plasma }, 0}$ is the initial plasma concentration of a bolus dose. $P$ is the permeability of the tumor vascular wall (8), and $R_{\text {cap }}$ is the radius of the capillary vessel. The remaining terms are the weighted average of the biexponential decay in the plasma. $\kappa$ and $\lambda$ are the normal capillary permeability and lymphatic drainage constants, respectively.

\section{Thiele Modulus}

The Thiele modulus captures the rate of antibody catabolism in the tumor compared with the time required to saturate the tissue by diffusion. If the characteristic time for internalization and catabolism is faster than the time required for antibody to reach the most distant tumor tissue $(\phi>1)$, antibody will be degraded before it completely saturates the tumor.

$$
\begin{aligned}
\phi_{\text {tumor }}^{2} & \equiv \frac{k_{e} R^{2}\left([A g]_{t} / \varepsilon\right)}{D\left(\frac{[A b]_{\text {plasma }}}{1+1 / B i}\right)} \\
& =\frac{\text { Antibody extravasation and diffusion time }}{\text { Antibody catabolism time }}
\end{aligned}
$$

where

$$
\begin{gathered}
B i \equiv \frac{2 P R_{\text {capillary }}}{D}=\frac{\text { Extravasation rate }}{\text { Diffusion rate }} \\
\phi_{m e t}^{2}=\frac{k_{e} R^{2}\left([A g]_{t} / \varepsilon\right)}{D[A b]_{n 0}}
\end{gathered}
$$

where the normal tissue concentration is

$$
[A b]_{n 0}=\frac{\kappa}{\lambda}[A b]_{\text {plasma }, 0},
$$

for a constant infusion and is set to the plasma concentration for bolus doses. The internalization rate constant $\mathrm{k}_{\mathrm{e}}$ represents the rate of catabolism in the tumor tissue, and $[A g]$ represents the concentration of internalizing antigen. $B i$ is the mass transfer Biot number, a dimensionless number characterizing transport resistances in series. For a small Biot number, permeability dominates over diffusion.

These parameters together define necessary conditions for tumor saturation:

$$
\phi^{2}<1 \quad \text { and } \quad \Gamma<1
$$

Failure of either criterion is sufficient for failure to saturate the tumor volume. In addition to providing criteria for saturation of a given tumor volume, $\phi_{\text {tumor }}^{2}$ and $\Gamma_{\text {tumor }}$ enable prediction of the depth of penetration of bound antibody. One of these parameters will approximately equal 1 at the distance of maximum antibody penetration, because by definition that is the point at which the diffusion or extravasation rate is balanced by either systemic clearance or antibody catabolism as illustrated in Figure 2.

\section{RESULTS}

For experimental validation (Fig. 3), 2 datasets that were not compounded by clearance effects were chosen to verify the Thiele modulus. Independent estimates for the various parameters were obtained from the literature (Tables 1 and 2 ). In the first experiment (10), single-chain variable fragments (scFvs) were dosed in anephric mice, eliminating rapid renal clearance. Before kidney removal, the clearance modulus of 3.8 indicates poor targeting due to the rapid drop in plasma concentration. In the second (11), continuous dosing of a slow-clearing antibody kept the effective concentration high throughout the experiment. Caution must be taken when interpreting other targeting results of tumors with high catabolism rates using radioactive tracers. Additional factors, such as the residualizing or nonresidualizing nature of the radioisotope, must also be taken into consideration (12). Also, for very low doses of radioactive antibodies used in diagnostics, more targeting may occur near the periphery of the tumor where there is some convection due to fluid leakage. By improving the moduli and saturating a larger fraction of the tumor, the imaging signal will 


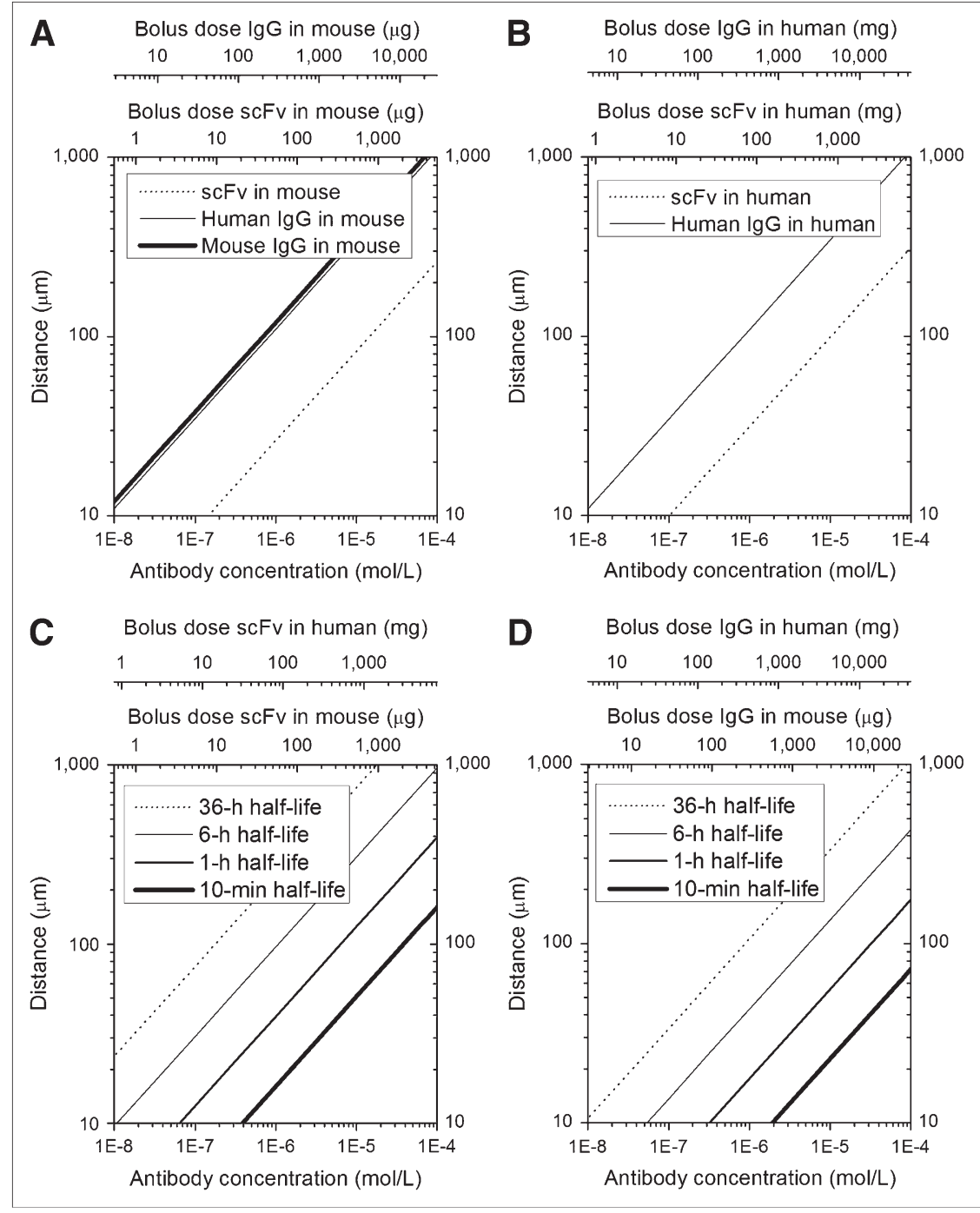

FIGURE 2. Antibody penetration distance limited by clearance (A and $\mathrm{B}$ ) or antigen turnover (C and $\mathrm{D})$. Pharmacokinetic parameters are from Table 1; antigen concentration is $0.1 \mu \mathrm{M}$. Formulas for $R$ are located in the supplemental data (available online only at http://jnm. snmjournals.org). be improved. However, this must be carefully weighed against any increased background from larger doses or longer clearance times.

The 2 criteria for effective targeting are not independent. In many cases, both clearance and internalization occur on similar time scales. To address the interaction between the two, full numeric simulations were performed with both internalization and clearance (Fig. 4).

\section{DISCUSSION}

The moduli provide insight into improving targeting. Increases in antibody dose unambiguously improve both $\phi^{2}$ and $\Gamma$ with capillary permeability necessitating large doses for solid tumors. Practical concerns, such as toxicity, immunogenicity, and economics, set an upper limit to this parameter. Extending the plasma half-life minimizes $\Gamma$ for agents such as scFvs, which clear too quickly to target all cells. A more rapid diffusivity helps targeting in micrometastases, and this is most easily achieved by smaller molecules, often trading off with faster clearance. To increase penetration into the tumor, $k_{e}$ should be minimized by using "noninternalizing" antibodies, such as antibodies that are effectively recycled in the endosome, extracellular matrix proteins, shed antigen, trapped necrotic debris, or perhaps tight-junction antigens. However, drugs requiring antibody uptake (e.g., immunotoxins and chemotherapeutic conjugates) clearly cannot apply this strategy; the particular problems with immunotoxin uptake versus diffusion have been quantitatively considered previously (13). Antigen level plays a subtle role in trading off pharmacodynamics vs. micropharmacokinetics. High expression levels provide a large amount of target, but this can limit penetration into the tumor. Although ideal for imaging, therapeutic targeting can be significantly hindered by these levels. Once $[A g]$ is high enough to enable delivery of a cell-killing dose of drug to an individual cell, any further increases only serve to worsen the distribution of drug throughout the tumor because of proportional increases in $\phi^{2}$ and $\Gamma$. For highly potent cytotoxic payloads such as $\alpha$-particle-emitting radionuclides that kill cells with delivery of only 1-10 
FIGURE 3. Experimental validation. (A) Model prediction (top); image adapted from Adams et al. (9), with yellow fluorescence marking capillary, red fluorescence indicating antibody, and green circle indicating model-predicted distance for penetration without any fitted parameters $\left(\mathrm{k}_{\mathrm{on}}=7 \times 10^{5} \mathrm{M}^{-1} \mathrm{~s}^{-1}\right.$; other parameters are in Table 1) (center); and fluorescence vs. distance using ImageJ (bottom). (B) Tumor size as fraction of control vs. Thiele modulus (with trastuzumab), defined using 100- $\mu \mathrm{m}$ distance, which corresponds with targeting $95 \%$ of tumor tissue (9). (C) Growth attenuation vs. fraction of tumor that is predicted to be saturated by model. The distances given in Baish et al. (9) were used.

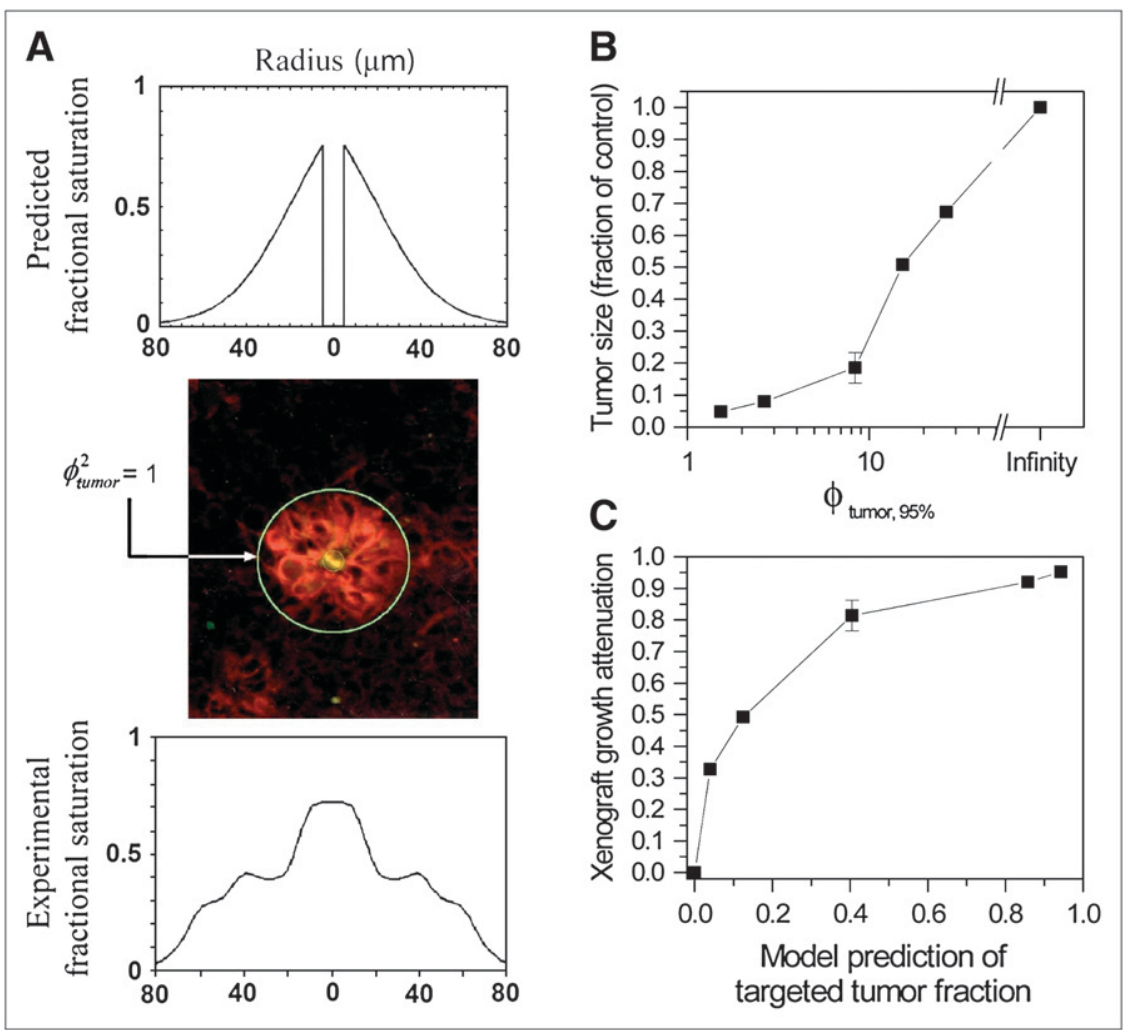

molecules of drug, antigens with $10^{5}-10^{6}$ molecules per cell present an unnecessarily onerous impediment to diffusion through the tumor tissue. Capillary permeability provides a very large barrier for solid tumor uptake as illustrated by the small Biot number. Attempts to improve $P$ in solid tumors through transcytosis by caveolar targeting have begun to meet with initial success in this regard (14). Even in these scenarios, it may not be possible to effectively target larger tumors with significant necrotic areas lacking functional blood vessels or even vascularized tumors less

TABLE 1

Previously Published Parameter Values Used in Simulations

\begin{tabular}{lll}
\hline Parameter & $\begin{array}{c}\text { Nephrectomized } \\
\text { mouse xenograft } \\
\text { (Adams et al.) }\end{array}$ & \multicolumn{1}{c}{$\begin{array}{c}\text { Multiple-dose } \\
\text { mouse xenograft } \\
\text { (Baselga et al.) }\end{array}$} \\
\hline $\mathrm{R}$ & $300 \mu \mathrm{m}$ & $100 \mu \mathrm{m}$ \\
$\mathrm{K}_{\mathrm{d}}$ & $15 \mathrm{pM}$ & $5 \mathrm{nM}$ \\
$\mathrm{R}_{\mathrm{cap}}$ & $5 \mu \mathrm{m}$ & $10 \mu \mathrm{m}$ \\
$\mathrm{D}$ & $80 \mu \mathrm{m}^{2} / \mathrm{s}$ & $14 \mu \mathrm{m}^{2} / \mathrm{s}$ \\
$\mathrm{P}$ & $5 \times 10^{-9} \mathrm{~m} / \mathrm{s}$ & $3 \times 10^{-9} \mathrm{~m} / \mathrm{s}$ \\
$\mathrm{Ag}$ & $0.15 \mu \mathrm{M}$ & $0.13 \mu \mathrm{M}$ \\
$\varepsilon$ & 0.3 & 0.1 \\
$\mathrm{Ab}$ & $2 \mu \mathrm{M}^{-4} / \mathrm{s}$ & $2.2 \times 10^{-4} \times 0.15=$ \\
$\mathrm{k}_{\text {endocytosis }}$ & $2.2 \times 10^{-4}$ & $3.3 \times 10^{-5} / \mathrm{s}$ \\
Biot number & $6 \times 10^{-4}$ & $4 \times 10^{-3}$
\end{tabular}

References for individual parameters are located in the supplemental data (available online only at http://jnm.snmjournals.org). than the roughly $1-\mathrm{cm}$ detection limit, which are slightly less heterogeneous. Finally, the distance $R$ from the circulating source of antibody drug will in general be smaller for micrometastases relative to vascularized bulk tumors, supporting a strategy of applying antibody therapeutics primarily to adjuvant therapy for minimal residual disease after resection (15).

\section{CONCLUSION}

Scaling arguments such as those presented in this paper can be useful analytic tools for qualitative consideration of overall systems behavior when multiple different rate phenomena are present (e.g., clearance, binding, endocytosis, diffusion). Arguably, the ability to generalize such simplistic analyses is actually preferable to detailed descriptive

TABLE 2

Clearance Parameters for Various Antibodies and Fragments

\begin{tabular}{lccc}
\hline $\begin{array}{c}\text { Whole-body pharmacokinetic } \\
\text { parameter }\end{array}$ & $\alpha$ & $\beta$ & $\% \alpha$ \\
\hline Mouse IgG in mouse & $1 \mathrm{~d}$ & $7 \mathrm{~d}$ & 70 \\
Human IgG in mouse & $1.2 \mathrm{~h}$ & $6.81 \mathrm{~d}$ & 66 \\
Human IgG in human & $12.74 \mathrm{~h}$ & $86.92 \mathrm{~h}$ & 43 \\
scFv in mouse & $0.05 \mathrm{~h}$ & $3 \mathrm{~h}$ & 80 \\
scFv in human & $0.42 \mathrm{~h}$ & $5.32 \mathrm{~h}$ & 90
\end{tabular}

References for individual parameters are located in the supplemental data (available online only at http://jnm.snmjournals.org). 


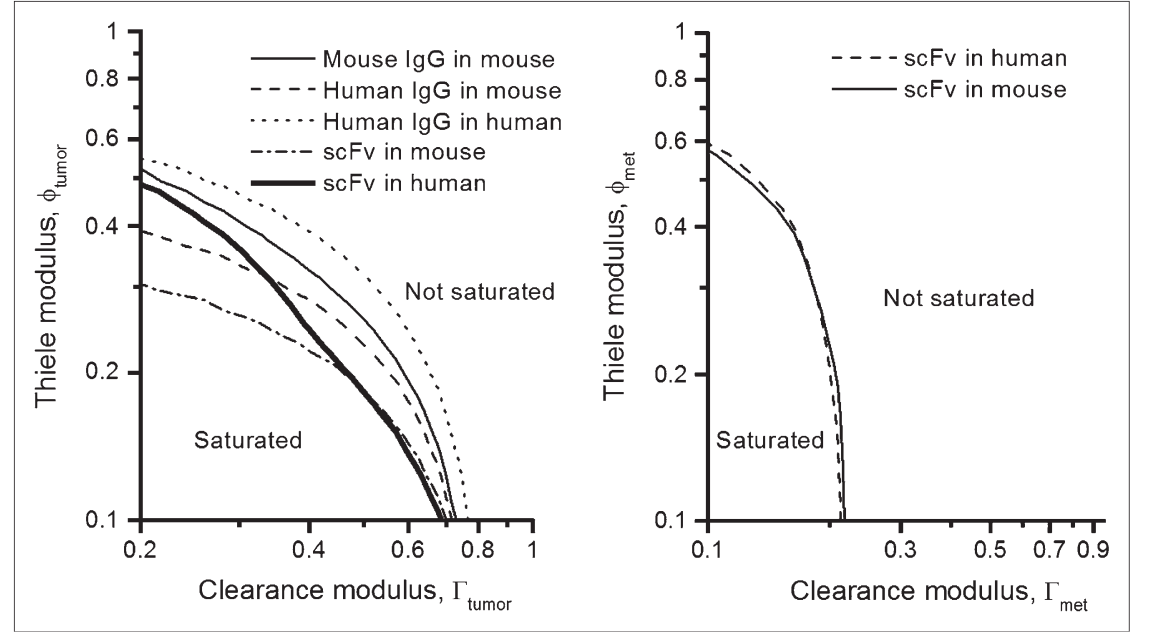

FIGURE 4. Full numeric simulations were performed with biexponential clearance and catabolism in tumor tissue. Antibody concentration was varied to change clearance modulus, and endocytosis rate was varied to independently change Thiele modulus. met $=$ metastasis. models of specific cases, when one is considering highly variable phenomena such as tumor physiology. The scaling analysis indicates that even low levels of antigen turnover can severely limit penetration of antibodies into tumor tissue; clearance of antibodies, especially fragments that are rapidly filtered, restricts penetration; and the large resistance of the tumor capillary wall may make antibodies more suitable for targeting micrometastases than solid tumors.

\section{ACKNOWLEDGMENTS}

Funding was provided by CA101830 and CA96504 and by a NIGMS/MIT Biotechnology Training Grant to one of the authors. Thanks are due to Matthew Lazzara for a detailed critical reading of the mathematic derivations and to Mike Schmidt for particularly helpful discussions of the significance of clearance versus penetration and antigen downregulation.

\section{REFERENCES}

1. Jain RK. Transport of molecules, particles, and cells in solid tumors. Annu Rev Biomed Eng. 1999;01:241-263.

2. van Osdol W, Fujimori K, Weinstein J. An analysis of monoclonal antibody distribution in microscopic tumor nodules: consequences of a "binding site barrier." Cancer Res. 1991;51:4776-4784.

3. Graff C, Wittrup KD. Theoretical analysis of antibody targeting of tumor spheroids: importance of dosage for penetration, and affinity for retention. Cancer Res. 2003;63:1288-1296.
4. Austin C, Maziere A, Pisacane P, et al. Endocytosis and sorting of ErbB2 and the site of action of cancer therapeutics trastuzumab and geldanamycin. Mol Biol Cell. 2004;15:5268-5282.

5. Baulida J, Kraus MH, Alimandi M, DiFiore PP, Carpenter G. All ErbB receptors other than the epidermal growth factor receptor are endocytosis impaired. J Biol Chem. 1996;271:5251-5257.

6. Kyriakos R, Shih L, Ong G, Patel K, Goldenberg D, Mattes MJ. The fate of antibodies bound to the surface of tumor cells in vitro. Cancer Res. 1992;52: 835-842.

7. Mattes MJ, Griffiths G, Diril H, Goldenberg D, Ong G, Shih L. Processing of antibody-radioisotope conjugates after binding to the surface of tumor cells. Cancer. 1994;73:787-793.

8. Gerlowski L, Jain RK. Microvascular permeability of normal and neoplastic tissues. Microvasc Res. 1986;31:288-305.

9. Baish J, Gazit Y, Berk D, Nozue M, Baxter L, Jain RK. Role of tumor vascular architecture in nutrient and drug delivery: an invasion percolation-based network model. Microvasc Res. 1996;51:327-346.

10. Adams G, Schier R, McCall A, et al. High affinity restricts the localization and tumor penetration of single-chain fv antibody molecules. Cancer Res. 2001;61: 4750-4755.

11. Baselga J, Norton L, Albanell J, Kim YM, Mendelsohn J. Recombinant humanized anti-HER2 antibody (Herceptin (TM)) enhances the antitumor activity of paclitaxel and doxorubicin against HER2/neu overexpressing human breast cancer xenografts. Cancer Res. 1998;58:2825-2831.

12. Press OW, Shan D, Howell Clark J, et al. Comparative metabolism and retention of iodine-125, yttrium-90, and indium-111 radioimmunoconjugates by cancer cells. Cancer Res. 1996;56:2123-2129.

13. Wenning LA, Murphy RM. Coupled cellular trafficking and diffusional limitations in delivery of immunotoxins to multicell tumor spheroids. Biotechnol Bioeng. 1999;62:562-575.

14. McIntosh D, Tan X, Oh P, Schnitzer J. Targeting endothelium and its dynamic caveolae for tissue-specific transcytosis in vivo: a pathway to overcome cell barriers to drug and gene delivery. Proc Natl Acad Sci U S A. 2002;99:1996-2001.

15. Koppe MJ, Bleichrodt RP, Oyen WJG, Boerman OC. Radioimmunotherapy and colorectal cancer. Br J Surg. 2005;92:264-276. 